\title{
EMBEDDING ANY COUNTABLE SEMIGROUP WITHOUT IDEMPOTENTS IN A 2-GENERATED SIMPLE SEMIGROUP WITHOUT IDEMPOTENTS
}

\author{
by KARL BYLEEN
}

(Received 2 June, 1986)

\begin{abstract}
Although the classes of regular simple semigroups and simple semigroups without idempotents are evidently at opposite ends of the spectrum of simple semigroups, their theories involve some interesting connections. Jones [5] has obtained analogues of the bicyclic semigroup for simple semigroups without idempotents. Megyesi and Pollák [7] have classified all combinatorial simple principal ideal semigroups on two generators, showing that all are homomorphic images of one such semigroup $P_{0}$ which has no idempotents.

In an earlier paper [1] a construction designed to produce regular simple semigroups was used to show that any countable semigroup can be embedded in a 2-generated bisimple monoid. In this paper a modification of the earlier construction is employed to prove that any countable semigroup without idempotents can be embedded in a 2-generated simple semigroup without idempotents, and to produce certain 2-generated congruence-free semigroups.

The reader is referred to the survey paper by Hall [3] and to Chapter 4 of Lyndon and Schupp [6] for discussions of related embedding theorems for semigroups, inverse semigroups, and groups. These references also contain extensive bibliographies. Clifford and Preston [2] or Howie [4] may be consulted for standard definitions and results from the theory of semigroups.
\end{abstract}

The author thanks Peter R. Jones for many helpful discussions.

1. The construction. Let $(S, \cdot)$ be any semigroup and let $A$ and $B$ be nonempty sets which are disjoint from each other and from $S$. Let $\alpha: A \times S \rightarrow A$ be a right action of $S$ on $A$ (i.e., $a \triangleright(s \cdot t)=(a \triangleright s) \triangleright t$ for all $a \in A$ and $s, t \in S$, where $a \triangleright s$ denotes $(a, s) \alpha)$. Let $\beta: S \times B \rightarrow B$ be a left action of $S$ on $B$ (i.e., $(s \cdot t) \triangleleft b=s \triangleleft(t \triangleleft b)$ for all $b \in B$ and $s, t \in S$, where $s \triangleleft b$ denotes $(s, b) \beta)$. Later in the paper juxtaposition will be used to denote the binary operation of $S$ as well as the right and left action, but in this section we use the more complicated notation in order to reserve juxtaposition to denote multiplication in the free semigroup $W^{+}$on the set $W=A \cup B \cup S$. Let $P=\left(p_{a b}\right)$ be an $A \times B$ matrix over $W$ such that $p_{a \triangleright s, b}=p_{a, s \triangleleft b}$ for all $a \in A, b \in B, s \in S$. Let $\mathscr{C}(S ; \beta, \alpha ; P)$ denote the semigroup with presentation

$$
\left\langle W ; a b=p_{a b}, a s=a \triangleright s, s b=s \triangleleft b, s t=s \cdot t \forall a \in A, b \in B, s, t \in S\right\rangle .
$$

Let $\Lambda$ denote the identity of the free monoid $W^{*}$ generated by the set $W$. For any nonempty subset $X$ of $W$ let $X^{*}$ denote the submonoid of $W^{*}$ generated by $X$, and let $X^{+}$ denote $X^{*} \backslash\{\Lambda\}$.

Glasgow Math. J. 30 (1988) 121-128. 
LEMMA 1.1. The elements of $\mathscr{C}(S ; \beta, \alpha ; P)$ are uniquely represented by the words in the set $R=B^{+} A^{*} \cup B^{*} S A^{*} \cup B^{*} A^{+}$.

Proof. The proof is conceptually the same as that of Lemma 1.1 of [1]. Define a mapping ${ }^{-}: W^{+} \rightarrow R$ by induction on the length $|w|$ of $w \in W^{+}$as follows: let $\bar{w}=w$ if $|w|=1$. If $w=w_{1} w_{2} \ldots w_{n+1}$ with each $w_{i} \in W$ and $n \geq 1$, denote $\overline{w_{1} w_{2} \ldots w_{n}}$ by $v t u$ where $v \in B^{*}, t \in S \cup\{\Lambda\}$, and $u \in A^{*}$;

$$
\begin{aligned}
& \text { if } \quad w_{n+1}=a \in A, \text { let } \bar{w}=v t u a \\
& \text { if } \quad w_{n+1}=s \in S, \quad \text { let } \quad \bar{w}= \begin{cases}v s & \text { if } u=t=\Lambda, \\
v(t \cdot s) & \text { if } u=\Lambda, t \neq \Lambda, \\
v t \hat{u}(a \triangleright s) & \text { if } u=\hat{u} a \neq \Lambda \text { where } \hat{u} \in A^{*}, a \in A ;\end{cases}
\end{aligned}
$$

and if

$$
w_{n+1}=b \in B, \quad \text { let } \quad \bar{w}= \begin{cases}v b & \text { if } u=t=\Lambda \\ v(t \triangleleft b) & \text { if } u=\Lambda, t \neq \Lambda, \\ \frac{v t \hat{u} p_{a b}}{4} & \text { if } u=\hat{u} a \neq \Lambda \text { where } \hat{u} \in A^{*}, a \in A\end{cases}
$$

This inductive definition establishes that any element $w \in W^{+}$may be reduced by the defining relations to an element $\bar{w} \in R$, and that $|\bar{w}| \leq|w|$.

To complete the proof of the lemma we show that no two distinct reduced words represent the same element of $\mathscr{C}(S ; \beta, \alpha ; P)$. Let $\psi: W \rightarrow \mathscr{T}_{R}$ be the mapping from $W$ into the full transformation semigroup on $R$ defined by $(v t u) x \psi=\overline{v t u x}$. This mapping extends to a semigroup homomorphism from $W^{+}$into $\mathscr{T}_{R}$. We use the definition of the mapping ${ }^{-}: W^{+} \rightarrow R$ to check that each of the four types of defining relations for $\mathscr{C}(S ; \beta, \alpha ; P)$ is satisfied in $\mathscr{T}_{R}$ by the elements of $W \psi$.

(1) $(v r u)[(a \psi)(b \psi)]=\overline{v r u a}(b \psi)=(v r u a) b \psi=\overline{v r u p_{a b}}=(v r u)\left(p_{a b} \psi\right)$, so $(a \psi)(b \psi)=$ $p_{a b} \psi$.

(2) $(v r u)[(a \psi)(s \psi)]=\overline{v r u a}(s \psi)=(v r u a)(s \psi)=v r u(a \triangleright s)=(v r u)(a \triangleright s) \psi$, so $(a \psi)(s \psi)=(a \triangleright s) \psi$.

(3) If $u=r=\Lambda$, then $(v r u)[(s \psi)(b \psi)]=(v s)(b \psi)=v(s \triangleleft b)=(v r u)(s \triangleleft b) \psi$, so $(s \psi)(b \psi)=(s \triangleleft b) \psi$. If $u=\Lambda, \quad r \neq \Lambda$, then $(v r u)[(s \psi)(b \psi)]=[v(r \cdot s)](b \psi)=$ $v((r \cdot s) \triangleleft b)=v(r \triangleleft(s \triangleleft b))=(v r)(s \triangleleft b) \psi$, so $(s \psi)(b \psi)=(s \triangleleft b) \psi$. If $u=\hat{u} a \neq \Lambda$, then $(v r u)[(s \psi)(b \psi)]=(v r \hat{u}(a \triangleright s)) b \psi=\overline{v r \hat{u} p_{a \triangleright s, b}}=\overline{v r \hat{p} p_{a, s \triangleleft b}}=(v r \hat{a} a)(s \triangleleft b) \psi$, so $(s \psi)(b \psi)=$ $(s \triangleleft b) \psi$.

(4) If $u=r=\Lambda$, then $(v r u)[(s \psi)(t \psi)]=(v s)(t \psi)=v(s \cdot t)=(v r u)(s \cdot t) \psi$, so $(s \psi)(t \psi)=(s \cdot t) \psi$. If $u=\Lambda, r \neq \Lambda$, then $(v r u)[(s \psi)(t \psi)]=[v(r \cdot s)](t \psi)=v((r \cdot s) \cdot t)=$ $v(r \cdot(s \cdot t))=(v r)(s \cdot t) \psi$, so $(s \psi)(t \psi)=(s \cdot t) \psi$. If $u=\hat{u} a \neq \Lambda$, then $(v r u)[(s \psi)(t \psi)]=$ $[v r \hat{u}(a \triangleright s)](t \psi)=v r \hat{u}((a \triangleright s) \triangleright t)=v r \hat{u}(a \triangleright(s \cdot t))=(v r \hat{u} a)(s \cdot t) \psi$, so $(s \psi)(t \psi)=(s \cdot t) \psi$.

Therefore the homomorphism $\psi$ factors through $\mathscr{C}(S ; \beta, \alpha ; P)$ giving a representation of $\mathscr{C}(S ; \beta, \alpha ; P)$ in $\mathscr{T}_{R}$. Let $b \in B$. If $v t u, y r x \in R$ and $(v t u) \psi=(y r x) \psi$, then 
$b[(v t u) \psi]=b[(y r x) \psi]$, so bvtu $=b y r x$ and thus $v t u=y r x$. Therefore $\psi$ is faithful and the uniqueness in the statement of the lemma is established.

As in the proof of Lemma 1.1 we will often denote a typical element of $R$ by $v t u$, where it is understood that $v \in B^{*}, t \in S \cup\{\Lambda\}, u \in A^{*}$ and $v t u \neq \Lambda$. Since each element of $S$ belongs to $R$ we have the following result.

Proposition 1.2. The semigroup $S$ is embedded in the semigroup $\mathscr{C}(S ; \beta, \alpha ; P)$.

Proposition 1.3. If $\Lambda \neq v u \in B^{*} A^{*}$, then $\overline{u v} \in A^{+} \cup B^{+} \cup S$.

Proof. The proposition is established by straightforward induction on the length of $v u$.

The construction of the semigroup $\mathscr{C}(S ; \beta, \alpha ; P)$ from the semigroup $S$ has a monoid version in which a monoid $\mathscr{C}(M ; \beta, \alpha ; P)$ is constructed from a monoid $(M, \cdot)$. In that case we require $\alpha: A \times M \rightarrow A$ and $\beta: M \times B \rightarrow B$ to be right and left monoid actions, respectively, i.e., we also require $a \triangleright 1=a$ and $1 \varangle b=b$ for all $a \in A, b \in B$. The matrix $P$ is an $A \times B$ matrix over $A \cup B \cup M$ such that $p_{a \triangleright m, b}=p_{a, m \triangleleft b}$ for all $a \in A, b \in B, m \in M$. Then $\mathscr{C}(M ; \beta, \alpha ; P)$ denotes the monoid with presentation

$$
\begin{aligned}
& \begin{array}{l}
\left\langle\cup \cup B \cup M ; a b=p_{a b}, a m=a \triangleright m, m b=m \triangleleft b, m n=\right. \\
\end{array} m . n, 1=\Lambda \\
&\forall a \in A, b \in B, m, n \in M\rangle .
\end{aligned}
$$

By the method of proof of Lemma 1.1 it can be shown that the elements of the monoid $\mathscr{C}(M ; \beta, \alpha ; P)$ are uniquely represented by the words in the set $B^{*} M A^{*}$, or equivalently, by the words in the set $\{\Lambda\} \cup B^{+} A^{*} \cup B^{*} \hat{M} A^{*} \cup B^{*} A^{+}$, where $\hat{M}$ denotes the set of non-identity elements of the monoid $M$.

The relationship between the semigroup and monoid versions of our construction is clarified by the following remarks. Suppose $S$ is any semigroup and $M$ is the monoid obtained from $S$ by adjoining a new identity element. If $\alpha$ and $\beta$ are right and left semigroup actions of $S$ on $A$ and $B$, respectively, let $\alpha^{\prime}$ and $\beta^{\prime}$ denote the right and left monoid actions of $M$ on $A$ and $B$ defined in the obvious way so as to extend $\alpha$ and $\beta$. Then the monoid obtained from $\mathscr{C}(S ; \beta, \alpha ; P)$ by adjoining a new identity element is isomorphic to $\mathscr{C}\left(M ; \beta^{\prime}, \alpha^{\prime} ; P\right)$ (this follows from Lemma 1.1 and its analogue for monoids referred to above).

In [1] the notation $\mathscr{C}(M ; B, A ; P)$ was used to denote the monoid $\mathscr{C}(M ; \beta, \alpha ; P)$ where $\alpha$ and $\beta$ are the trivial right and left actions defined by $a \triangleright m=a$ and $m \triangleleft b=b$ for all $a \in A, b \in B, m \in M$.

2. The embedding. By choosing the actions $\alpha$ and $\beta$ and the matrix $P$ appropriately the semigroup $\mathscr{C}(S ; \beta, \alpha ; P)$ can be guaranteed to have certain nice properties. We show how the result of the title may be obtained in this way. Suppose first that $S, \alpha, \beta, P$ are as in Lemma 1.1.

Proposition 2.1. If the semigroup $S$ is idempotent-free, then so is $\mathscr{C}(S ; \beta, \alpha ; P)$. 
Proof. Suppose $y=y^{2}$ in $\mathscr{C}(S ; \beta, \alpha ; P)$. Let $y=v t u \in R$. Then $t \neq \Lambda$, for otherwise $\overline{v u v u}=v u$, which is impossible since by Proposition $1.3 \overline{u v} \in A^{+} \cup B^{+} \cup S$. Thus $v t u v t u=v t u$, so $\overline{u v} \in S$ and $t(\overline{u v}) t=t$ which implies that $t(\overline{u v})$ is an idempotent of $S$.

LemMA 2.2. If $\alpha$ and $\beta$ are trivial, each row and column of $P$ contains an element of $S$, and the entries of $P$ which belong to $S$ generate $S$, then $\mathscr{C}(S ; \beta, \alpha ; P)$ is simple.

Proof. Let $u s u, w t x \in \mathscr{C}(S ; \beta, \alpha ; P)$. If $v \neq \Lambda$ then since each column of $P$ contains an element of $S$ there exists $u^{\prime} \in A^{+}$such that $u^{\prime} v \in S$. If $v=\Lambda$ let $u^{\prime}=\Lambda$. Similarly, if $u \neq \Lambda$ there exists $v^{\prime} \in B^{+}$such that $u v^{\prime} \in S$. If $u=\Lambda$ let $v^{\prime}=\Lambda$. Suppose that $t \neq \Lambda$. Then since the entries of $P$ which belong to $S$ generate $S$ we have $t=p_{a_{1}, b_{1}} p_{a_{2}, b_{2}} \ldots p_{a_{k}, b_{k}}$ where $a_{i} \in A, \quad b_{i} \in B$ for $i=1,2, \ldots, k$. Hence in $\mathscr{C}(S ; \beta, \alpha ; P)$ we have $w a_{1} u^{\prime}(v s u) v^{\prime} b_{1} a_{2} b_{2} \ldots a_{k} b_{k} x=w a_{1} r b_{1} a_{2} b_{2} \ldots a_{k} b_{k} x=w a_{1} b_{1} a_{2} b_{2} \ldots a_{k} b_{k} x=w t x$ where $r \in S$. If $t=\Lambda$ then either $|w|>0$ or $|x|>0$. If $t=\Lambda$ and $|w|>0$ then $w=\hat{w} b$ for some $b \in B$. Then $\hat{w} u^{\prime}(v s u) v^{\prime} b x=\hat{w} r b x=\hat{w} b x=w t x$, where $r \in S$. If $t=\Lambda$ and $|x|>0$ then $x=a \hat{x}$ for some $a \in A$. Then $w a u^{\prime}(v s u) v^{\prime} \hat{x}=w a r \hat{x}=w a \hat{x}=w t x$, where $r \in S$. In all cases $w t x \leq, v s u$, hence $\mathscr{C}(S ; \beta, \alpha ; P)$ is simple.

THEOREM 2.3. Any semigroup without idempotents can be embedded in a simple semigroup without idempotents.

Proof. Let $S$ be an idempotent-free semigroup. Let $A=\{a\}$ and $B=\left\{b_{s}: s \in S\right\}$ be sets disjoint from each other and from $S$, where $A$ is a singleton and $B$ is a set in one-to-one correspondence with $S$, and let $P$ be the $A \times B$ matrix such that $p_{a, b_{s}}=s$ for all $s \in S$. Let $\alpha$ and $\beta$ be the trivial actions. Then $\mathscr{C}(S ; \beta, \alpha ; P)$ contains $S$ by Proposition 1.2, is idempotent-free by Proposition 2.1, and is simple by Lemma 2.2 .

THEOREM 2.4. Any countable semigroup without idempotents can be embedded in a 2-generated simple semigroup without idempotents.

Proof. Let $S$ be any countable semigroup without idempotents, and let $A=$ $\left\{a_{1}, a_{2}, a_{3}, \ldots\right\}$ and $B=\left\{b_{1}, b_{2}, b_{3}, \ldots\right\}$ be countably infinite sets disjoint from each other and from $S$. Let $P$ be an $A \times B$ matrix over $A \cup B \cup S$ such that (1) $p_{n n}=a_{n+1}$ and $p_{n+1, n}=b_{n+1}$ for $n=1,2,3, \ldots$, (2) each row and column of $P$ contains an element of $S$, and (3) each element of $S$ appears somewhere in $P$. Let $\alpha$ and $\beta$ be the trivial actions. Then $S$ is embedded in $\mathscr{C}(S ; \beta, \alpha ; P)$ by Proposition 1.2 , and $\mathscr{C}(S ; \beta, \alpha ; P)$ is idempotent-free by Proposition 2.1 and is simple by Lemma 2.2. Furthermore, since $a_{n} b_{n}=p_{n n}=a_{n+1}$ and $a_{n+1} b_{n}=p_{n+1, n}=b_{n+1}$ for $n=1,2,3, \ldots$ each element of $A \cup B$ is generated by $a_{1}$ and $b_{1}$. But each element of $S$ appears as a product $a_{i} b_{j}=p_{i j}$, so $A \cup B \cup S$ and thus $\mathscr{C}(S ; \beta, \alpha ; P)$ is generated by $a_{1}$ and $b_{1}$.

The following result shows that Theorem 2.4 cannot be strengthened by replacing "simple" by "bisimple".

Proposition 2.5. Any finitely generated bisimple semigroup is regular.

Proof. Let $S$ be a finitely generated bisimple semigroup. Since $S$ is bisimple either (1) $S$ has a non-trivial $\mathscr{R}$-class, or (2) $S$ has a non-trivial $\mathscr{L}$-class, or (3) $S$ is trivial. 
Suppose that (1) holds. Then every $\mathscr{R}$-class is non-trivial and $x \in x S$ for each $x \in S$. Since $S$ is finitely generated there exists a maximal $\mathscr{L}$-class $L_{a}$ of $S$. Since $a \in a S$ we have $a=a s$ for some $s \in S$. But this implies $L_{a} \leq L_{s}$, so $a \mathscr{L}_{s}$ and thus $s=t a$ for some $t \in S^{1}$. Hence $a=a t a$, and thus $S$ is regular. The argument is dual if (2) holds, while the result is trivial if (3) holds.

Baer-Levi semigroups and Croisot-Tessier semigroups [2] are examples of right simple semigroups without idempotents. Proposition 2.5 implies that such semigroups cannot be finitely generated.

3. An example. Theorem 2.4 would be strengthened if "simple" could be replaced by "congruence-free". We do not know whether any countable semigroup (without idempotents) can be embedded in a 2-generated congruence-free semigroup (without idempotents), but we show that our construction can be used to provide an example of a 2-generated congruence-free semigroup without idempotents. This semigroup is of course simple, but by Proposition 2.5 is not bisimple.

Let $S=\langle s\rangle$ be the infinite cyclic semigroup, let $A=\left\{a_{1}, a_{2}, a_{3}, \ldots\right\}$ and $B=$ $\left\{b_{1}, b_{2}, b_{3}, \ldots\right\}$ be countably infinite sets disjoint from each other and from $S$, and let $\alpha: A \times S \rightarrow A$ and $\beta: S \times B \rightarrow B$ be the actions of $S$ on $A$ and $B$ defined by $a_{i} s^{k}=a_{i+k}$, $s^{k} b_{j}=b_{j+k}$ for $i, j, k=1,2,3, \ldots$ Let $P$ be an $A \times B$ matrix over $A \cup B \cup S$ such that

(P1) $p_{11}=s$;

(P2) $p_{1+i, j}=p_{i, j+1}$ for $i, j=1,2,3, \ldots$;

(P3) given $x, y \in\left\{a_{1}, b_{1}, s\right\}$ and distinct positive integers $i, j$ there exists a positive integer $k$ such that $p_{i k}=x=p_{k i}$ and $p_{j k}=y=p_{k j}$.

We note that (P2) guarantees the condition $p_{a s, b}=p_{a, s b}$ of our construction, and now show that a matrix $P$ satisfying (P1)-(P3) exists. Once the first row of $P$ is specified, the entire matrix is uniquely determined by (P2). One way to specify the first row of $P$ so that (P1) and (P3) are satisfied is as follows: for $n=0,1,2, \ldots$ let $K_{n}=\left\{x s^{n} y: x, y \in\right.$ $\left.\left\{a_{1}, b_{1}, s\right\}\right\}$. Thus $K_{n}$ consists of 9 words from $\left\{a_{1}, b_{1}, s\right\}^{+}$. Let $w_{n}$ be the concatenation of the words in $K_{n}$ and let $w$ be the $\omega$-word defined by $w=s w_{0} w_{1} w_{0} w_{1} w_{2} w_{0} w_{1} w_{2} w_{3} w_{0} \ldots$ Then any word in any $K_{n}$ appears infinitely often as a subword of $w$. Let $p_{1 n}$ be the $n$th letter of $w$. Then (P1) is satisfied. We check that (P3) is satisfied. Let $i, j$ be distinct positive integers and let $x, y \in\left\{a_{1}, b_{1}, s\right\}$. Without loss of generality we may assume that $i<j$. Let $r=j-i$. Then $x s^{r-1} y \in K_{r-1}$ so there exists a positive integer $m>i$ such that $p_{1, m}=x, p_{1, m+r}=y$. Hence by $(\mathrm{P} 2)$ we have $p_{m-i+1, i}=x=p_{i, m-i+1}$ and $p_{j, m-i+1}=y=$ $p_{m-i+1, j}$, which establishes (P3).

Proposition 3.1. $\mathscr{C}(\langle s\rangle ; \beta, \alpha ; P)$ is a 2-generated congruence-free semigroup without idempotents.

Proof. By (P1) we have $a_{1} b_{1}=s$, so by the actions of $S$ on $A$ and $B$ we conclude that $A \cup B \cup S$ and thus $\mathscr{C}(\langle s\rangle ; \beta, \alpha ; P)$ is generated by $a_{1}$ and $b_{1}$. By Proposition 2.1 $\mathscr{C}(\langle s\rangle ; \beta, \alpha ; P)$ is idempotent-free.

To show that $\mathscr{C}(\langle s\rangle ; \beta, \alpha ; P)$ is congruence-free it suffices to show that if $\rho$ is a congruence on $\mathscr{C}(\langle s\rangle ; \beta, \alpha ; P)$ and if $v s^{m} u, y s^{r} x$ are distinct elements of $\mathscr{C}(\langle s\rangle ; \beta, \alpha ; P)$ 
such that $v s^{m} u \rho y s^{r} x$, then $\rho=\omega$, the universal congruence. We prove this by considering several cases depending on the forms of the elements $v s^{m} u$ and $y s^{r} x$.

Case 1. Suppose $v=y=s^{m}=s^{r}=\Lambda$. We prove that $\rho=\omega$ by induction on $|u|$. As the basis of the induction suppose $|u|=1$, so $u=a_{i}$ for some $i$. We now use induction on $|x|$. If $|x|=1$, then $x=a_{j}$ for some $j \neq i$ and $a_{i} \rho a_{j}$. By (P3) there exists a positive integer $k$ such that $a_{i} b_{k}=s, a_{j} b_{k}=a_{1}$. Again by (P3) there exists a positive integer $l$ such that $a_{i} b_{l}=s, a_{j} b_{l}=b_{1}$. Hence $s \rho a_{1}$ and $s \rho b_{1}$. Multiplying the first relation on the right by $s$, and the second on the left by $a_{1}$, yields $s^{2} \rho a_{2} \rho s$, and so every element of $\mathscr{C}(\langle s\rangle ; \beta, \alpha ; P)$ is in the idempotent congruence class which contains $s, a_{1}, b_{1}$. So $\rho=\omega$. Now suppose $|x|>1$, say $x=\hat{x} a_{j}$ where $\hat{x} \in A^{+}$. If $i \neq j$ then by (P3) there exists $k$ such that $a_{i} b_{k}=a_{1}$, $a_{j} b_{k}=s$, so $a_{1} \rho \hat{x} s$ where $|\hat{x} s|<|x|$ and $\hat{x} s \neq a_{1}$; so by induction $\rho=\omega$. If $i=j$ then by (P3) there exists $k$ such that $a_{i} b_{k}=s$, so $s \rho \hat{x} s$. Let $\hat{x}=\hat{x} a_{l}$ where $\hat{x} \in A^{*}$. Then multiplication on the left by $a_{l+1}$ yields $a_{l+2} \rho a_{l+1} \hat{x} a_{l+1}$, so by the case $i \neq j$ we conclude $\rho=\omega$.

Now as the induction hypothesis for induction on $|u|$ we suppose that if $|u|=n$ and $u \rho x, u \neq x$, then $\rho=\omega$. Let $|u|=n+1$, say $u=\hat{u} a_{i}, x=\hat{x} a_{j}$. If $i \neq j$ then by (P3) there exists $k$ such that $a_{i} b_{k}=s, a_{j} b_{k}=a_{1}$, so $\hat{u} s \hat{x} a_{1}$, and by induction $\rho=\omega$. If $i=j$, then by (P3) there exists $l$ such that $a_{i} b_{l}=s, a_{j} b_{l}=s$, so $\hat{u} s \hat{x} s$ and again $\rho=\omega$.

Case 2. Suppose $u=x=s^{m}=s^{r}=\Lambda$. This case is dual to Case 1 , so $\rho=\omega$.

Case 3. Suppose $v \neq y$. (i) If $u \neq \Lambda, x \neq \Lambda$, then multiplying $v s^{m} u \rho y s^{r} x$ on the right by appropriate elements of $B$ and using (P3) we conclude that $v s^{m} a_{1} \rho y s^{r} a_{1}$; so $v s^{m} b_{1} \rho y s^{r} b_{1}$ and thus $v b_{m+1} \rho y b_{r+1}$. By Case $2 \rho=\omega$. (ii) If $u=x=\Lambda$, then again $v s^{m} b_{1} \rho y s^{\prime} b_{1}$ so $\rho=\omega$. (iii) If $u=\Lambda, x \neq \Lambda$, then $v s^{m} \rho y s^{r} x$ yields $v s^{m} z \rho y s^{r} a_{1}$ by multiplication on the right by an appropriate $z \in B^{+}$. There exist $k$ and $l$ such that $a_{1} b_{k}=a_{1}$ and $a_{1} b_{l}=b_{1}$. Thus $v s^{m} z b_{k}^{|y|+1} b_{l} \rho y s^{r} b_{1}$, so by Case 2, $\rho=\omega$.

Case 4. Suppose $u \neq x$. This case is dual to Case 3 , so $\rho=\omega$.

Case 5. Suppose $v=y$ and $u=x$. Then $m \neq r$ and $v s^{m} u=y s^{r} x$. If $v=y=\Lambda$, then multiplication on the left by $a_{1}$ yields $a_{m+1} u \rho a_{r+1} x$, so $\rho=\omega$ by Case 1 . If $v=y \neq \Lambda$, then multiplication on the left by an appropriate element of $A^{+}$yields $a_{1} s^{m} u \rho a_{1} s^{r} x$, so again $\rho=\omega$ by Case 1 .

4. Congruence-free monoids. The first four cases of the proof of Proposition 3.1 can be adapted with only slight modifications (the induction in Case 1 , for example, starts with $|u|=0$ ) to prove Proposition 4.1 below. Let $M$ be any monoid, let $A=$ $\left\{a_{1}, a_{2}, a_{3}, \ldots\right\}$ and $B=\left\{b_{1}, b_{2}, b_{3}, \ldots\right\}$ be countably infinite sets disjoint from each other and from $M$, let $\alpha$ and $\beta$ be the trivial actions of $M$ on $A$ and $B$, respectively, and let $P^{\prime}$ be an $A \times B$ matrix over $A \cup B \cup M$ which satisfies

(P1) ${ }^{\prime} p_{n, n}=a_{n+1}, p_{n+1, n}=b_{n+1}$ for $n=1,2,3, \ldots$;

(P2) those entries of $P^{\prime}$ which belong to $M$ generate $M$; and

(P3) given $x, y \in\left\{1, a_{1}, b_{1}\right\}$ and distinct positive integers $i, j$ there exists a positive integer $k$ such that $p_{i k}=x=p_{k i}$ and $p_{j k}=y=p_{k j}$. 
Then conditions $(\mathrm{P} 1)^{\prime}$ and $(\mathrm{P} 2)^{\prime}$ imply that the monoid $\mathscr{C}\left(M ; B, A ; P^{\prime}\right)$ is generated by $a_{1}$ and $b_{1}$.

Proposition 4.1. Let $\rho$ be a proper congruence on the monoid $\mathscr{C}\left(M ; B, A ; P^{\prime}\right)$, with $A, B, M, P^{\prime}$ as above. Then vsupytx implies $v=y$ and $u=x$.

Proposition 4.2. If $M$ is a congruence-free monoid and $\rho$ is a proper congruence on $\mathscr{C}\left(M ; B, A ; P^{\prime}\right)$ with $A, B, M, P^{\prime}$ as above, then either $\rho$ is the identity or $\rho=$ $\left\{(v s u, v t u): s, t \in M, u \in A^{*}, v \in B^{*}\right\}$.

Proof. Suppose $\rho$ is a proper congruence on $\mathscr{C}\left(M ; B, A ; P^{\prime}\right)$. Since $M$ is congruencefree the restriction $\rho \mid M$ is either the identity $i d_{M}$ or the universal congruence $\omega_{M}$. Suppose $\rho \mid M=i d_{M}$. If $v s u \rho y t x$, then by Proposition 4.1 we have $v=y$ and $u=x$. Therefore, by multiplication on right and left, $s \rho t$ so $s=t$. Hence $\rho$ is the identity. Suppose $\rho \mid M=\omega_{M}$. Then $\left\{(v s u, v t u): s, t \in M, u \in A^{*}, v \in B^{*}\right\} \subseteq \rho$, while by Proposition 4.1 the reverse inclusion holds. Hence $\rho=\left\{(v s u, v t u): s, t \in M, u \in A^{*}, v \in B^{*}\right\}$.

Proposition 4.3. Any countable semigroup can be embedded in a 2-generated bisimple monoid which has exactly three congruences.

Proof. From Theorems 8.1 and 8.2 (and the remarks on p. 234) of [6] any countable semigroup can be embedded in a countable algebraically closed monoid $M$, and any algebraically closed monoid is congruence-free. A monoid $N$ is bisimple if and only if for each $a \in N$ the equations $x w=a, y a=w, w z=1$ can be solved by elements $w, x, y, z \in N$. Since any monoid can be embedded in a bisimple monoid (this follows from Preston's embedding [9] of any semigroup into a bisimple monoid, and the fact that local submonoids of a bisimple regular semigroup are isomorphic), any algebraically closed monoid must be bisimple (that algebraically closed semigroups are bisimple is noted in [8]). Thus the monoid $\mathscr{C}\left(M ; B, A ; P^{\prime}\right)$, with $A, B, P^{\prime}$ as above, is bisimple by Theorem 2.4 of [1], is generated by $a_{1}$ and $b_{1}$, and by Proposition 4.2 (provided $M$ is non-trivial) has exactly 3 congruences.

We note that if $M$ is trivial, then Proposition 4.2 implies that any proper congruence is the identity, and so $\mathscr{C}\left(M ; B, A ; P^{\prime}\right)$ is a congruence-free monoid generated by $a_{1}$ and $b_{1}$.

In conclusion we pose some questions suggested by our results. The first asks for an analogue for semigroups of the theorem that any countable group can be embedded in a 2-generated simple group (see p. 190 of [6]).

1. Can any countable semigroup be embedded in a 2 -generated (finitely generated) congruence-free semigroup?

2. Can any countable semigroup without idempotents be embedded in a 2-generated (finitely generated) congruence-free semigroup without idempotents?

3. Does there exist a bisimple semigroup which is neither left simple, nor right simple, nor regular?

\section{REFERENCES}

1. K. Byleen, Embedding any countable semigroup in a 2-generated bisimple monoid, Glasgow Math. J. 25 (1984), 153-161. 
2. A. H. Clifford and G. B. Preston, The algebraic theory of semigroups, (Amer. Math. Soc. 1961, 1967).

3. T. E. Hall, Inverse and regular semigroups and amalgamation: a brief survey, Proceedings of a Symposium on Regular Semigroups, Northern Illinois University (1979), pages 49-79.

4. J. M. Howie, An introduction to semigroup theory, (Academic Press, 1976).

5. P. R. Jones, Analogs of the bicyclic semigroup in simple semigroups without idempotents.

6. R. C. Lyndon and P. E. Schupp, Combinatorial group theory, (Springer-Verlag, 1977).

7. L. Megyesi and G. Pollák, On simple principal ideal semigroups. Studia Sci. Math. Hung. 16 (1981), 437-448.

8. B. H. Neumann, Algebraically closed semigroups, in Studies in Pure Mathematics, (Academic Press, 1971) pp. 185-194.

9. G. B. Preston, Embedding any semigroup in a $\mathscr{D}$-simple semigroup, Trans. Amer. Math. Soc. 93 (1959), 351-355.

Department of Mathematics, Statistics and Computer Science

Marquette University

MilwaukeE

WISCONSIN 53233, USA 